\title{
FPGA-Based Configurable Systolic Architecture for Window-Based Image Processing
}

\author{
César Torres-Huitzil \\ Computer Science Department, National Institute for Astrophysics, Optics and Electronics, P.O. Box 51 and 216, 72000 Puebla, Mexico \\ Email:ctorres@inaoep.mx
}

Miguel Arias-Estrada

Computer Science Department, National Institute for Astrophysics, Optics and Electronics, P.O. Box 51 and 216, 72000 Puebla, Mexico Email: ariasm@inaoep.mx

Received 13 September 2003; Revised 21 May 2004

\begin{abstract}
Image processing requires more computational power and data throughput than most conventional processors can provide. Designing specific hardware can improve execution time and achieve better performance per unit of silicon area. A fieldprogrammable-gate-array- (FPGA-) based configurable systolic architecture specially tailored for real-time window-based image operations is presented in this paper. The architecture is based on a $2 \mathrm{D}$ systolic array of $7 \times 7$ configurable window processors. The architecture was implemented on an FPGA to execute algorithms with window sizes up to $7 \times 7$, but the design is scalable to cover larger window sizes if required. The architecture reaches a throughput of $3.16 \mathrm{GOPs}$ at a $60 \mathrm{MHz}$ clock frequency and a processing time of 8.35 milliseconds for $7 \times 7$ generic window-based operators on $512 \times 512$ gray-level images. The architecture compares favorably with other architectures in terms of performance and hardware utilization. Theoretical and experimental results are presented to demonstrate the architecture effectiveness.
\end{abstract}

Keywords and phrases: FPGA, configurable system, real time, window-based image processing, systolic array.

\section{INTRODUCTION}

Designing a hardware processor core for data-intensive image processing is a fundamental step in developing modern machine vision systems that can efficiently implement computer vision related tasks. The wide interest in dataintensive or window-based image processing is due to the fact that more complex algorithms use the low-level results as primitives to pursue higher-level goals [1]. However, building such systems remains difficult because of three main reasons [2]. First, most computer vision applications are computational intensive tasks difficult to overcome using conventional processors. The sequential nature of conventional processors and the huge amount of redundant data involved in an image slow down the performance of vision systems. Second, heterogeneity of computations in the processing performed through an application limits the parallelization. A mismatch, therefore, exists between the complexity of the required operations and the processing power offered by processors. Third, several vision applications require systems with real-time performance. A real-time system is one that must interact with its environment under responsetime constraints $[3,4]$. Real-time image processing systems interact with their environment, and thus they must produce outputs that are not only numerically correct, but which also meet timing constraints necessary for these interactions.

On the other hand, advances in computer technology have made the building of machine vision systems a tractable problem, resulting in a widespread use of these systems in applications such as robotics, multimedia, virtual reality, industrial inspection, medical engineering, and autonomous navigation. However, processing power, physical size, and power consumption constraints restrict further the extent to which these applications are solved under the perspective of systems on a chip [5].

Previously, there have been several architectures proposed in the literature to implement window-based image processing algorithms $[6,7,8]$. However, almost all of them focus on the performance of a single image processing algorithm, usually 2D image convolution, without considering implementation aspects such as flexibility, silicon area, and power consumption.

In this paper, we propose an improved 2D systolic architecture as an alternative solution to implement windowbased image processing algorithms on a single chip with a good performance/area tradeoff. The rest of the paper is 


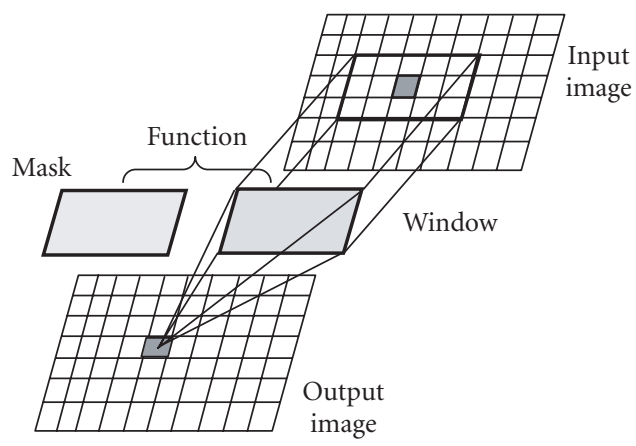

FIGURE 1: Conceptual view of a window-based operator.

organized as follows. Section 2 describes the basis of window-based image processing. The systolic architecture organization and the processing element structure are described in Section 3. Section 4 presents the complexity and performance issues of the architectural model. Experimental results and the performance analysis of the FPGA implementation are presented in Section 5. Discussion and comparison are given in Section 6. Finally, conclusions and further work are presented in Section 7.

\section{WINDOW-BASED IMAGE PROCESSING}

The first stages of computer vision require a large number of repetitive neighbor or window-based operations over a massive amount of image data. The window-based operation domain is restricted to a small neighbor of image data centered on a reference pixel. A conceptual view of window-based operators is depicted in Figure 1. A window-based operation is performed when a window, area of $w \times w$ pixels, is extracted from the input image and it is transformed according to a window mask or kernel and mathematical functions to produce an output result $[1,9,10]$. The window mask is the same size as the image window and their values are usually constants through the entire image processing. The values used in the window mask depend on the specific type of features to be detected or recognized. Usually a single output data is produced by each window operation and it is stored in the same position as the central pixel of the window.

Window-based operations can be formalized mathematically as follows. Let $I$ be the $M \times N$ input image, $Y$ the output image, and $W$ a $w \times w$ window mask. A $2 \mathrm{D}$ window operation can be defined as

$$
Y_{r c}=F\left(f\left(w_{i j}, I_{r+i, c+j}\right)\right) \quad \forall(i, j) \in w \times w, \forall(r, c) \in M \times N,
$$

where $w_{i j}$ represents a coefficient from the window mask $W$, $I_{r+i, c+j}$ represents a pixel from a $w \times w$ window around the $(r, c)$ pixel in the input image, $f$ defines a scalar function, and $a$ defines the local reduction function.

Window-based operators are characterized because the same scalar function is applied on a pixel by pixel way to each individual pixel of one or more input images to produce a partial result. Common scalar functions include relational operations, arithmetic operations, logical operations,
TABLE 1: Complexity of image convolution expressed in terms of arithmetic operations.

\begin{tabular}{lc}
\hline Elemental operations & Number of executions \\
\hline Multiplication & $w^{2} \times M \times N$ \\
Addition & $\left(w^{2}-1\right) \times M \times N$ \\
Load/store & $\left(2 \times w^{2}+1\right) \times M \times N$ \\
\hline
\end{tabular}

and possibly look-up tables. The local reduction function reduces the window of intermediate results, computed by the scalar function, to a single output result. Some common local reduction functions employed are accumulation, maximum, and absolute value. Scalar and local reduction functions form the image algebra to construct window-based image applications $[9,11]$. Thus, to design flexible hardware architectures for window-based image processing, the architecture instruction set must support these functions.

\subsection{Computational requirements}

Window-based image operations in computer vision are computationally expensive tasks. The window-based operation complexity can be expressed in terms of the elementary arithmetic operations required to process an image [8]. In particular, analyzing image convolution, the total number of operations required to perform this task, excluding address generation and other control actions, is summarized in Table 1 . The values presented in Table 1 were obtained considering the convolution of a $w \times w$ window mask on an $M \times N$ input image. In general terms, a window-based operator has a computational complexity of $O\left(w^{2} \times M \times N\right)$. This complexity can be reduced for special cases such as separable filters, but the general case is considered for explanation purposes.

Window-based image processing also involves a high data transfer rate (DTR) between the image acquisition module, the image memory, and the processor. If a binary representation of $b$ bits for pixels and an image processing rate of $f_{f}$ images per second, the input data transfer rate is given by

$$
\mathrm{DTR}_{I}=b \times(2 \times w \times w) \times M \times N \times f_{F} .
$$

In a similar way, the output data transfer rate is given by

$$
\operatorname{DTR}_{O}=b \times M \times N \times f_{F} .
$$

The high data transfer rates and computational complexity involved in window-based image processing require an efficient use of communication channel bandwidth and the use of parallel processing to achieve high processing efficiencies.

\subsection{Implementation issues}

Data-intensive image processing algorithms require the input image memory to be accessed several times. This is particularly true for window-based operations. Considering the gap between a processor speed and memory access time, 
the memory access time overhead is a critical issue to face. Although window-based image operations may access memory in different patterns, they share important features that can be generalized as follows:

(i) window-based image operations are memory intensive, at least one new pixel of data is typically needed for each step in the computation,

(ii) a high potential for parallelism is available since window operations include a large percentage of independent operations that are applied to each pixel or region of a large $2 \mathrm{D}$ image array.

On the other hand, image data is usually stored in linear memory organizations so that neighboring pixels in the image are not necessarily stored as neighboring elements in linear memories. This obscures the spatial relationship between the pixels so the $2 \mathrm{D}$ data parallelism is hidden. If the spatial data dependencies are exposed, it should be easier to implement a hardware architecture to use uniform 2D data access patterns that are needed for parallel execution. A related issue is that data for window operators usually overlaps with the neighbor windows of the pixels surrounding. This means that there is a great deal to create simple vectors of data elements that can be processed by parallel vectorization techniques $[6,8,12]$. However, introducing a high degree of parallelism increases the requirements of internal local storage and communication routing. Thus a compromise among performance and silicon area should be chosen according to a given application. However, the exposed drawbacks can be partially solved using an adequate memory scan to reduce the memory access time and exploit data parallelism through parallel architectures [8]. The memory addressing used in this work to exploit parallelism is explained in Section 2.3.

A key factor to take into account to implement a hardware architecture is the implementation technology. In this research, the field programmable gate array (FPGA) technology was chosen due to the following characteristics $[13,14]$ :

(i) FPGA provides massive parallel structures and high density for logic arithmetic,

(ii) in FPGA devices, tasks are implemented by spatially composing primitive operators rather than temporally,

(iii) in FPGAs, it is possible to control operations at bit level to build specialized datapaths,

(iv) FPGA technology can offer potentially two orders of magnitude more raw computational power per unit of area than conventional processors with shorter design cycles compared to ASICs,

(v) FPGA technology is well suited for implementing parallel architectures such as SIMD-like and systolic processors.

\subsection{Data parallelism and memory addressing}

In this section, a technique for extracting the 2D spatial data dependencies from window-based image processing is presented. It is based on a previous work reported in [15] where
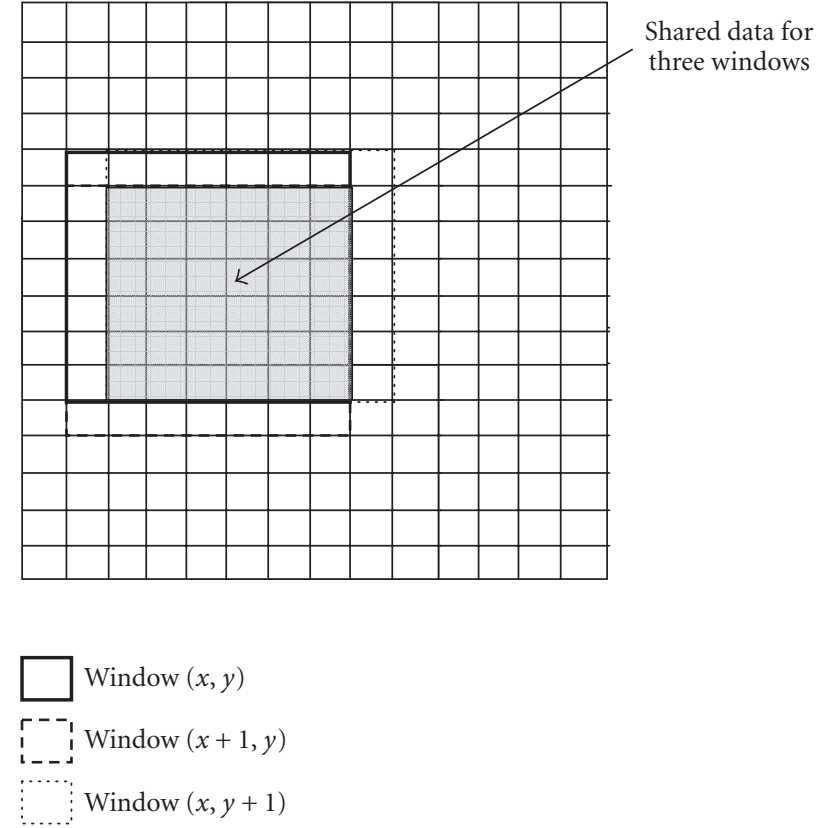

Figure 2: Data-level parallelism in window-based image operations.

an efficient image addressing mode was used in an architecture for fast edge and corner detection. In this work the addressing mode is generalized and extended for a generic window-based operator and the design of a new architecture is proposed. In Figure 2, a set of three windows is depicted on an input image. The center of two windows are shifted, one column and one row, from a reference window centered at position $(x, y)$. The enclosed area by each window represents the number of pixels required to produce an output data. As can be seen from Figure 2, some data is overlapped among the three windows. This implies that some pixels could be used for the computation of three window operations in the same reading cycle of the input memory diminishing the number of memory accesses by a factor of three on the shared-data area. The current read pixel could be broadcast to different processors in order to compute several window operators in parallel.

The idea exposed above can be extended for a greater number of windows in the input image in a close neighbor where data sharing can be exploited. The larger number of windows considered in a close neighborhood, the less the data overlapping. A criterion should be defined in order to establish a good compromise between data overlapping and the number of window operations performed in parallel.

The inherent data parallelism in window-based image operators can be combined with loop unrolling in order to diminish the memory access overhead of a purely sequential implementation.

A traditional sequential implementation of a $w \times w$ window-based operation would slide the window horizontally across the image until it reaches the end of a given row, then moves one row below and repeat the process. Under this 


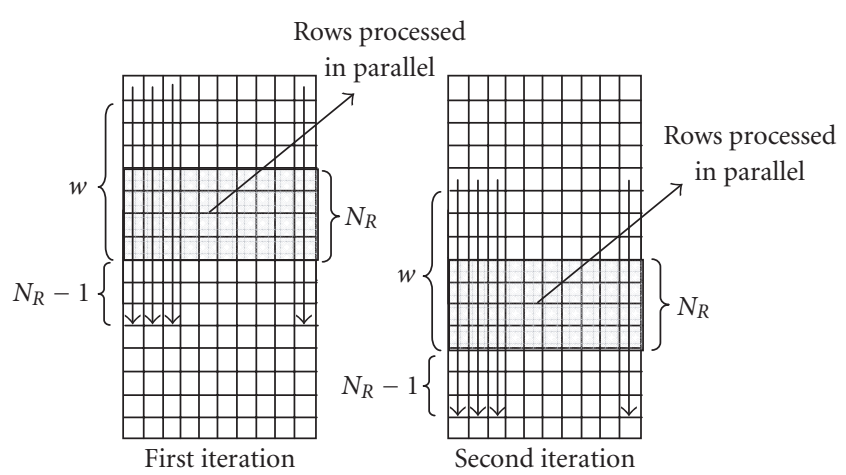

Figure 3: Column-based image addressing mode to exploit data parallelism and loop unrolling.

approach, every pixel in the image is read several times. The sequential memory time overhead can be diminished by partially unrolling the loop and computing more than one window in parallel either in the vertical or in the horizontal direction. Loop unrolling is a process of replacing the iterations of a loop with noniterated straight-line operations $[8,16]$. For the purpose of explanation, partial loop unrolling in the vertical direction is considered but the horizontal direction can be used interchangeably. The use of partial unrolling in the vertical direction combined with an adequate addressing scheme allows the processing of several rows in parallel.

The addressing mode used in this work is based on a column-based order as shown in Figure 3. The number of pixels read per column is directly dependent on the degree of loop unrolling employed, that is, the number of rows processed in parallel. If $N_{R}$ rows are processed in parallel over an $M \times N$ image and a $w \times w$ window mask, then the number of pixels read per column is $w+N_{R}-1$. Figure 3 shows two iterations of the image processing when $N_{R}$ rows are processed in parallel. In the first iteration $N_{R}$ rows are being processed in concurrently and when their processing is complete, then the processing of another set of $N_{R}$ rows starts.

Under the proposed addressing scheme, the total number of memory accesses $N_{\mathrm{MA}}$ to process the image is given by

$$
N_{\mathrm{MA}}=N \times\left(w+N_{R}-1\right) \times\left[\frac{M}{N_{R}}\right],
$$

where $M \times N$ is the image dimension, $w$ the window size, and $N_{R}$ the number of rows processed in parallel.

The term in parentheses in (4) represents the number of pixels read per column and the term in brackets represents the number of strips that the input image is divided into to process several rows in parallel. According to (4), the number of memory accesses depends almost linearly on the window size in contrast to the quadratic dependence in the sequential implementation of a window operator. In fact, the dependence tends asymptotically to be proportional with a unit constant as the number of rows processed in parallel increases.

\section{SYSTOLIC ARCHITECTURE}

In this section some details of the proposed hardware architecture for window-based image processing are presented. The architecture exploits data parallelism and reuse through a column-based memory addressing mode and a set of processing elements working in parallel. The computation core of the architecture is organized under a $2 \mathrm{D}$ systolic array of processing elements. The processing element specially designed for the systolic architecture is called configurable window processor (CWP).

The architecture is a stand-alone processor, but the memories for storage of the input image and the processed image are external to the architecture. The architecture reads data from the input memory banks where input image pixels and window mask coefficients are stored, denoted as $P$ and $W$, respectively. The image pixels are read in a column-based scan and they are transmitted to the array of processing elements to compute, in parallel, several window operations.

\subsection{Array of configurable window processors}

The array of processing elements is the computation core of the architecture. The processing elements are arranged under a 2D systolic approach. Figure 4 shows the 2D systolic organization of the CWPs. The 2D systolic array extends the concurrency of the architecture to $2 \mathrm{D}$ parallelism, that is, the parallel computation of window operations through rows and columns on the input image. In Figure 4, the module denoted by CWP represents a configurable window processor, the module labeled by $\mathrm{D}$ represents a delay line or shift register, and the module labeled with LDC represents a local data collector. For each column of the systolic array there is a local data collector that collects results of CWPs located in the same column of the array. The global data collector module collects the results and sends them to the output memory. The CWPs in the same column, enclosed by a rectangle, compute a window operator on the same column of the input image. This set of CWPs can be considered as a 1D systolic array. Data flows from top to bottom, and the CWPs work progressively in a systolic pipeline. The control module and the address generator unit are not shown in Figure 4 for simplicity.

A delay line is required in the boundary of the CWPs in a column in order to support partial unrolling. The delay line is designed as a serial-input serial-output shift register. Thus, the window coefficients are temporally stored in the delay line, delayed for synchronization purposes, and then transmitted to the next column of CWPs. The stage number in the delay line depends on the loop unrolling degree employed. If $N_{R}$ rows are processed in parallel, then a delay line with $N_{R}-1$ register is required because of a mismatch between the window mask size and the number of pixels read per column. In other words, the number of clock cycles required to all the coefficients of a column of the window mask pass throw a column of CWPs is $w+N_{R}$.

After $w+N_{R}$ clock cycles, the coefficients of the current column of the window mask are sent progressively to the next set of CWPs and the coefficients of another column of the 


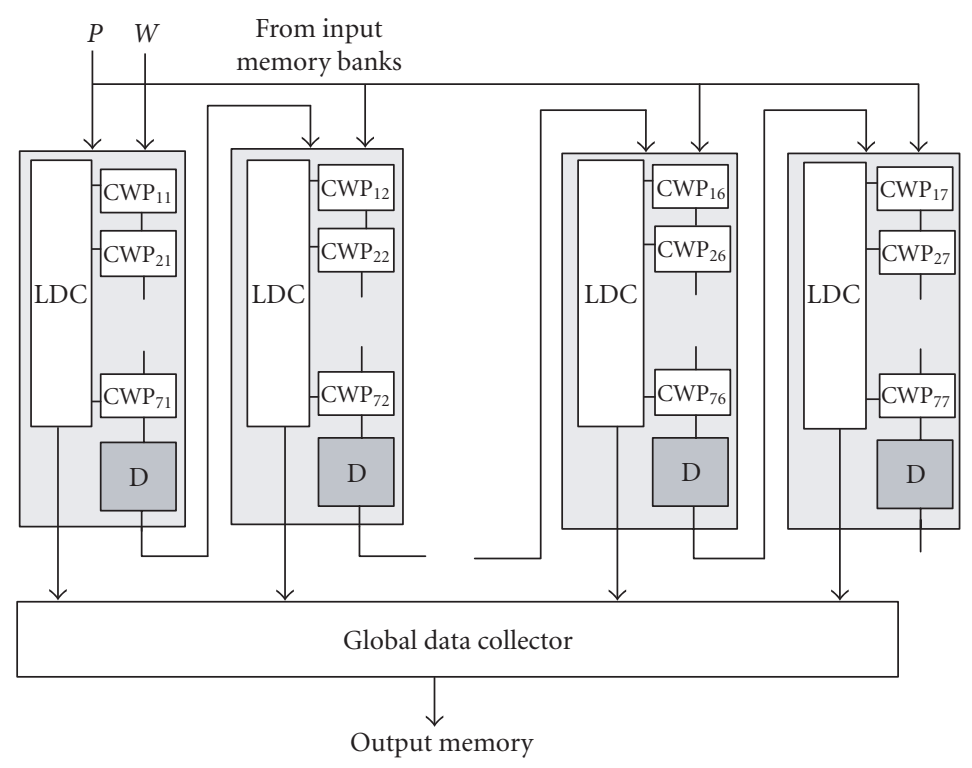

(a)

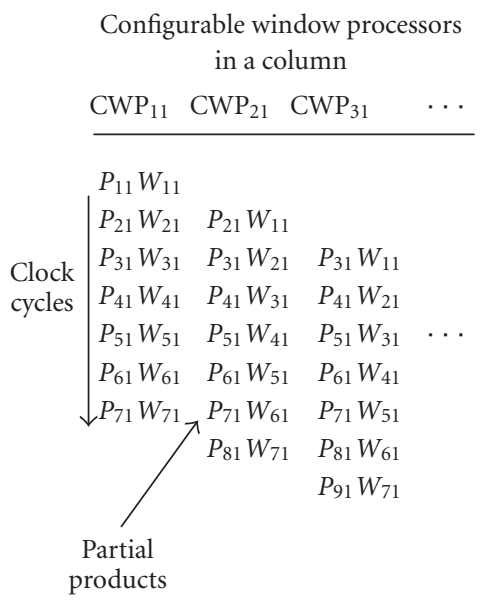

(b)

FIGURE 4: (a) Systolic array for fast speed computing of window-based image operations. (b) Data flow and computation for some CWPs in the same column for image convolution.

mask is fed to the current set of CWPs. A detail of the data flow of window mask coefficients through CWPs is shown in Figure 4.

The local data collector collects data from the CWPs, and the captured result is sent out of the CWPs to a global data collector. The local data collector scans progressively the CWPs from top to bottom according to a control signal from the control unit module in the architecture.

The interconnection of several columns of CWPs towards a 2D systolic array extends the concurrency of the architecture to $2 \mathrm{D}$ parallelism by exploiting data parallelism through parallel processing, pipeline, and loop unrolling. The interconnection between columns of CWPs is straightforward and it can be considered a cascaded connection of 1D systolic arrays. In the 2D array, data flows from top to bottom of a column, and then left to right through columns.

As a whole, the architecture operation starts when a pixel from the input image is broadcast to all the processing elements in the array. Each CWP working in parallel keeps track of a particular window-based operation. At each clock cycle, a CWP receives a different window coefficient, stored in an internal register, and an image pixel that is common for all the processing elements. These values are used by each CWP to carry out a computation, specified by a scalar function, and to produce a partial result of the window operation. The partial results are incrementally sent to the local reduction function implemented in the CWP to produce a single result when all the pixels of the window are processed. In Figure 4 a detail of the data flow and the computation steps for three CWPs in the same column of the systolic array is shown for several clock cycles when the systolic array is configured for image convolution with a $7 \times 7$ window mask. Each CWP, if enabled, performs the computation of a multiplication and an accumulation.

After a short latency period, each CWP has progressively computed an output of a window operation. The produced result is captured by the data collectors and stored in the output image memory. Once a result produced by a CWP is collected, the processing element is ready to start a new computation. The CWPs work progressively in the same manner until all data in the input image has been processed. The CWPs are continuously being reutilized for computing different windows in the input image.

\subsection{Configurable window processor}

Figure 5 shows a simplified block diagram of the processing element designed to cover most window-based operations in image processing. It is called a configurable window processor (CWP). The CWP is composed of a register, an arithmetic processor (AP), and a local reduction module (LRM). The AP is based on a similar scheme of an arithmetic logic unit (ALU) and provides the hardware support for the scalar functions. It includes a multiplier, an adder/subtracter, and a distance computation module. The LRM module implements the local reduction function and it includes an accumulator and a maximum/minimum computation module. For instance, for image convolution, the AP module performs a multiplication and the LRM module performs an accumulation. Similarly, for gray-level dilation/erosion, the AP module and LRM module perform additions/subtraction and maximum/minimum operations, respectively.

The CWP performs the computational work required in a given window-based application. The CWP is configurable by a control word selected by the end user. The CWP has two 


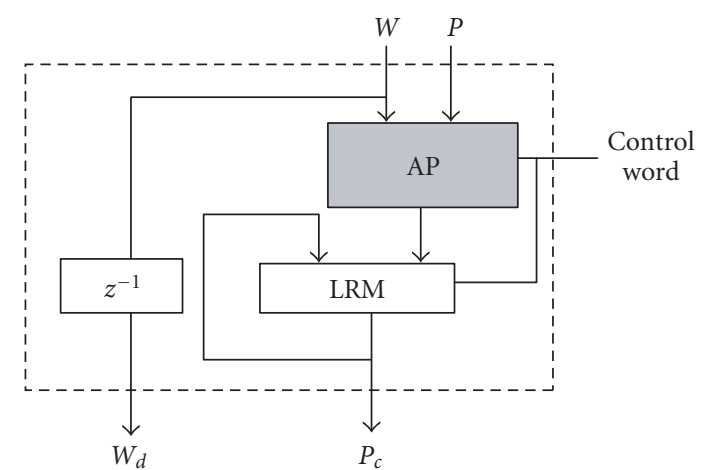

Figure 5: Block diagram of the configurable window processor.

operational inputs, pixels from the input image, and coefficients from the window mask, denoted by $P$ and $W$, respectively. Each CWP has two output signals. One of them is the result of the window operation $\left(P_{C}\right)$ and the other one is a delayed value of a window coefficient $\left(W_{d}\right)$ that is transmitted to its neighbor CWP or to a delay line. The CWP accepts one pixel data and a window coefficient to compute one step of a window operation in a clock cycle.

The functionality of the CWP is configured by a control word in run time. The user sends a control word through a port of the architecture to control the operation that the AP and LRM must perform according to an application.

In summary, on each clock cycle, each CWP executes in parallel three disjoint operations:

(i) compute the pixel by pixel value to be passed to the next computation cycle,

(ii) integrate the contents of the output register, calculated at the previous clock cycle, with the new value produced by the AP module,

(iii) read a new window coefficient and store it into the register then, transmit the previous coefficient to the next CWP.

\section{COMPLEXITY AND PERFORMANCE ISSUES}

The hardware architecture presented in the previous sections is dedicated to window-based image processing under realtime constraints. The main concerns for the architecture design were a regular input data scheme with a small number of data input/output ports, and fast parallel processing of window operations. In this section, some metrics are established to evaluate the complexity and performance of the architecture in term of architectural parameters. Throughout the analysis, it is considered that the proposed architecture consists of $N_{\mathrm{CWP}}(R)$ rows of configurable window processors. Each row includes $N_{\mathrm{CWP}}(C)$ configurable window processors.

\subsection{Processing time}

The required processing time to process an input image with a window-based operator is composed of two main times: the latency time and the parallel processing time.
The latency time corresponds to the time required to start pipeline operation inside the $2 \mathrm{D}$ systolic array. The latency time is measured between the activation of the first CWP until the activation of the last CWP. The latency is significant when the $2 \mathrm{D}$ systolic array starts the processing of a new set of rows of the input image.

The time required to initialize full pipeline operation of the parallel modules at the beginning of row processing is given by

$$
\begin{aligned}
\tau_{l}= & {\left[(\text { MaskSize }-1) \times\left(N_{\mathrm{CWP}}(R)+\text { MaskSize }-1\right)\right] } \\
& \times \frac{1}{F} \times \frac{M}{N_{\mathrm{CWP}}(R)},
\end{aligned}
$$

where $N_{\mathrm{CWP}}(R)$ is the number of rows processed in parallel, MaskSize is the size of mask used in the computations, $F$ is the main clock frequency, $M$ is the number of rows of the image, and $N$ is the number of columns of the image.

The parallel processing time is the time when all the CWPs in the $2 \mathrm{D}$ systolic array are working in parallel. This is the addition of all the computing times to process a set of rows, without considering the latency at the start of processing.

The required parallel processing time to process $M \times N$ pixels, without the initial latency is given by

$$
\tau_{p}=M \times N \times\left[\frac{N_{\mathrm{CWP}}(R)+\text { MaskSize }-1}{N_{\mathrm{CWP}}(R)}\right] \times \frac{1}{F} .
$$

The factor enclosed in brackets represents the average clock cycle number to produce a result. The overall time needed to process an $(M \times N)$-sized image is given by the summation of the preceding times:

$$
\mathrm{T}=\tau_{l}+\tau_{p}
$$

From the architectural point of view, the performance is compromised with the number of rows processed concurrently, hence with the silicon resources allocated to the architecture.

\subsection{Throughput}

The throughput of a system can be expressed in terms of the number of elemental operations that the system can perform per second. In this sense, only the operations contributing to the computation of a result are considered. In the particular case of the architecture, two operations are of significance, the scalar function and the local reduction function executed on each clock cycle. Under these considerations, the computational throughput of the architecture can be calculated according to the following equation:

$$
\text { Throughput }=F \times\left(N_{\mathrm{CWP}}(R) \times N_{\mathrm{CWP}}(C)\right) \times \mathrm{NIP}_{\mathrm{CWP}} \text {, }
$$

where $F$ is the main clock frequency, $N_{\mathrm{CWP}}(R) \times N_{\mathrm{CWP}}(C)$ the number of processing elements in the systolic array, and NIP $_{\text {CWP }}$ the average number of elemental operations computed per CWP on each clock cycle. 


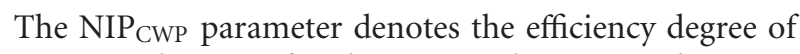
computational usage of each CWP in the 2D systolic array.

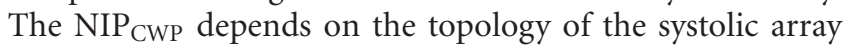
and the dimension of the problem to be solved. The NIP CWP $_{\text {}}$ can be computed according to the following equation:

$$
\mathrm{NIP}_{\mathrm{CWP}}=2 \times \frac{\text { MaskSize }}{N_{\mathrm{CWP}}(R)+\text { MaskSize }-1},
$$

where MaskSize denotes the dimension of the window used in the window-based image operator.

\section{RESULTS}

To achieve the required characteristics related to the architecture performance and configurability, a fully parameterizable VHDL description of the modules of the proposed architecture was carried out by making extensive use of generictype configurations inputs. Furthermore, a fully structural description of these modules was also carried out, using only the most elementary logic operations provided by the implementation library to achieve good optimization levels for the main components of the architecture. Since structural VHDL was used for a hierarchical design, the whole design is platform, version, technology, and tool independent.

The modules and the complete architecture were synthesized with the XST tool and placed and routed with Foundation ISE from Xilinx. All the simulations were carried out with Active HDL, both functional and postsynthesis simulations.

\subsection{Hardware resource utilization}

This section presents the FPGA synthesis results for the main modules of the architecture. The hardware resource utilization is reported for an XCV2000E-6 VirtexE FPGA device. The digital synthesis was carried out in a hierarchical and incremental way. The results are summarized in Table 2, using as main parameters the number of slices, and specifically the number of flip-flops and look-up tables of the FPGA.

The results shown in Table 2 were obtained with the XST synthesis tool configured to optimize for speed. A $7 \times 7$ systolic array of CWPs was synthesized. Since no temporal restrictions of low-level were imposed on the place and route process, further improvements in area and speed can be obtained if timing and area restrictions are imposed. The hardware resource utilization is only a small part of the FPGA resources, about 30 percent. In fact, the architecture suits well for FPGAs with lower logic capacities.

An available prototype of the architecture, synthesized for an XCV2000E-6 Virtex device, executes approximately 3.16 GOPs at a $60 \mathrm{MHz}$ clock frequency. The hardware architecture was prototyped using an RC1000PP FPGA-based board from Alpha Data. The FPGA-based board is centered on a VirtexE device with an equivalent logic capacity of 2 million gates. The board is attached to the PCI bus of a host computer, and it contains $8 \mathrm{MB}$ on-board memory organized in four banks of $2 \mathrm{MB}$ each. The memory and hardware resources available in the board can be accessed through an
TABLE 2: FPGA synthesis summary for the architecture and its main modules.

\begin{tabular}{lccc}
\hline \multicolumn{4}{c}{ XCV2000E-6 FPGA VirtexE device } \\
\hline Module & $\begin{array}{c}\text { Number of } \\
\text { slices }\end{array}$ & $\begin{array}{c}\text { 4-input } \\
\text { LUTs }\end{array}$ & $\begin{array}{c}\text { Number of } \\
\text { flip-flops }\end{array}$ \\
\hline $\begin{array}{l}\text { CWP } \\
\text { address } \\
\text { Generator } \\
\text { unit }\end{array}$ & 101 & 190 & 24 \\
$\begin{array}{l}\text { Control unit } \\
\text { A column of } \\
7 \text { CWPs }\end{array}$ & 154 & 269 & 97 \\
$\begin{array}{l}\text { Complete } \\
\text { architecture }\end{array}$ & 540 & 10 & 3 \\
\hline
\end{tabular}

TABle 3: Technical data for the systolic architecture for real-time window-based image processing. The architecture was synthesized for a $7 \times 7$ systolic array.

\begin{tabular}{ll}
\hline \multicolumn{2}{c}{$\begin{array}{c}\text { Technical data for the systolic architecture } \\
\text { XCV2000E-6 FPGA Virtex device }\end{array}$} \\
\hline Feature & Specification \\
\hline FPGA technology & $\begin{array}{l}0.18 \mu \mathrm{m} \text { 6-layer metal } \\
\text { process } \\
\text { Power supply }\end{array}$ \\
$\begin{array}{l}\text { Number of processing } \\
\text { elements, CWPs }\end{array}$ & 49 \\
$\begin{array}{l}\text { Clock frequency } \\
\text { FPGA percentage for the } \\
\text { complete architecture }\end{array}$ & $60 \mathrm{MHz}$ \\
$\begin{array}{l}\text { FPGA percentage per } \\
\text { CWP }\end{array}$ & 6118 slices, around 30\% \\
$\begin{array}{l}\text { FPGA percentage, address } \\
\text { and control logic }\end{array}$ & 101 slices, less than 1\% \\
$\begin{array}{l}\text { Number of flip-flops } \\
\text { Estimated power } \\
\text { consumption }\end{array}$ & 159 slices, less than 1\% \\
$\begin{array}{l}\text { Off-chip memory data } \\
\text { buses }\end{array}$ & 1604 \\
$\begin{array}{l}\text { On-chip memory } \\
\text { Peak performance }\end{array}$ & $1.56 \mathrm{~W}$ \\
Number of pins & 21 -bit address, 8-bit data \\
\hline
\end{tabular}

API developed in Visual C++. An experimental setup for architecture prototyping with real images was built using this board.

A summary of the technical data for the FPGA implementation of the current architecture prototype, highlighting aspects such as technology, hardware resources, performance, and power consumption, is shown in Table 3. The current prototype is based on a systolic array of $7 \times 7$ CWPs. The estimated power consumption of the architecture was obtained with the Xilinx XPower software tool using a clock frequency of $60 \mathrm{MHz}$. 


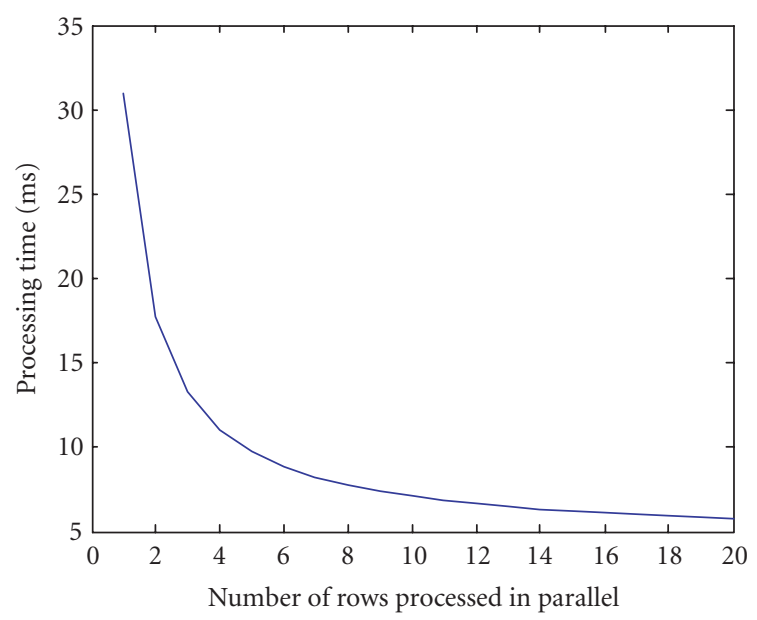

Figure 6: Architecture performance with different parallelism degrees.

\subsection{Performance}

In this section the results of the processing time of the proposed architecture for performing window-based operators on an input image are presented. The results and graphs were obtained using the synthesis results and the equations presented and discussed in Section 4.

Figure 6 shows the processing time of the architecture versus the number of rows processed in parallel. Per each row, a set of MaskSize CWPs is employed in the 2D systolic array. The processing time is the time required to process an image on the proposed architecture when image data is already available in the input image memory. All the additional times needed for the $I / O$ requests and data transfers are not considered in the performance analysis of the architecture since they are dependent on the prototyping platform. Thus, the image acquisition overhead must be added to the processing time. However, the image acquisition overhead can be overcome in a hardware prototyping platform if an appropriate double buffering is employed, that is, the acquisition process is performed concurrently with the processing using independent memory banks.

A $512 \times 512$ image and a $7 \times 7$ window mask were considered to plot the curves in Figure 6. An increase in the number of CWPs produces an increase in the overall performance of the architecture. As can be seen from Figure 6, a systolic array with the capability of processing two rows in parallel would be enough to fulfil real-time requirements, that is, processing of 30 frames per second.

The processing times for a window-based operation on $512 \times 512$ gray-level images using different window sizes are plotted in Figure 7 . The image processing time using a $7 \times 7$ window mask is 8.35 milliseconds, which leaves time for image acquisition or image memory loading since about 30 milliseconds are required to achieve standard real-time constraints. Moreover post-processing on the resulting image can be applied. The performance results for different window sizes are plotted on the same figure. The 2D systolic array was

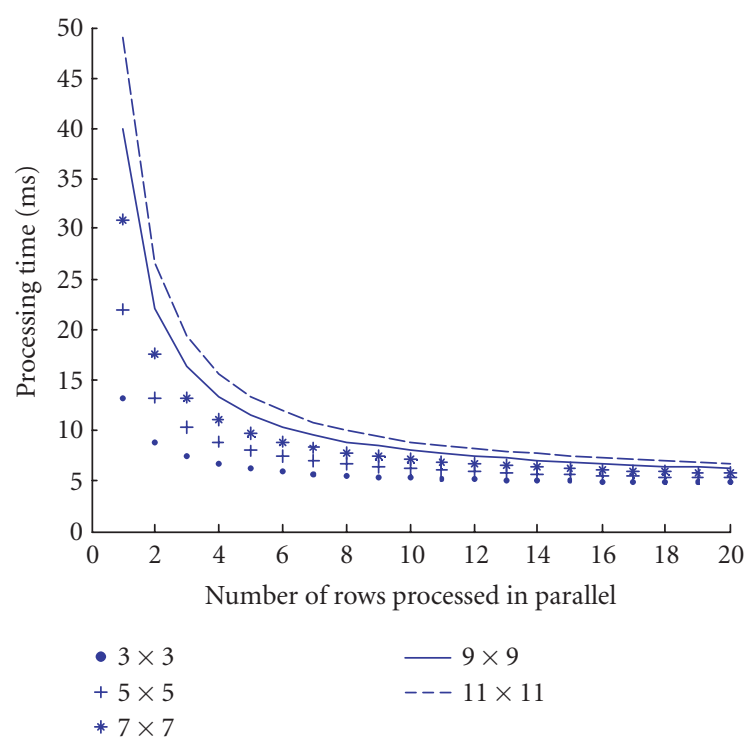

Figure 7: Performance of the proposed architecture for a $512 \times 512$ gray-level image with different window sizes where the array has an equal dimension to window mask.

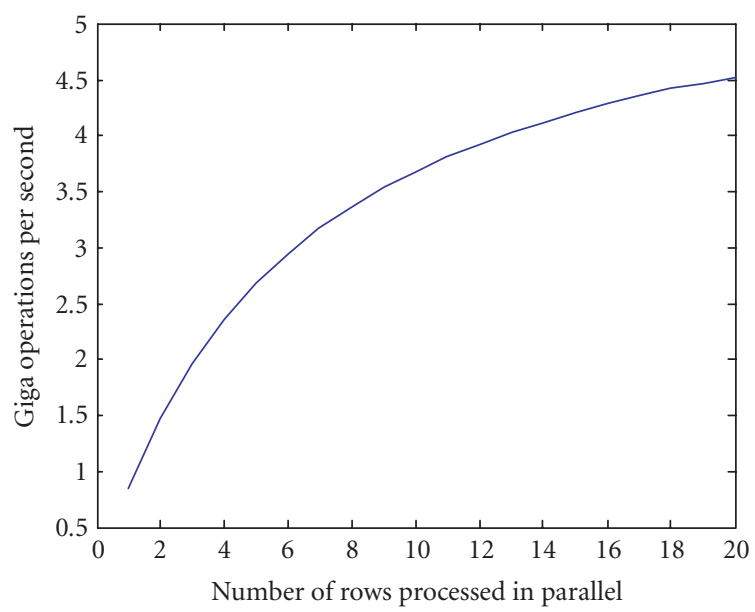

Figure 8: Throughput of the architecture for different configurations of the 2D systolic array.

configured to use the same number of processing elements as the window size. For all the cases, it is possible to achieve realtime performance with low hardware resource utilization.

Figure 8 shows the achievable throughput of the architecture when different parallelism degrees, number of processing elements, are employed. The graph shows the number of GOPs versus the processing elements employed. As can be seen, the throughput increases with the number of processing elements. However, if many processing elements are used in the systolic array the utilization and activity efficiency of the processing elements decrease. Thus, an appropriate reutilization scheme should be investigated to increase the computational density at the expense of an increase in the control logic. 
A performance improvement can be obtained-either optimizing the design mapped onto the FPGA or employing an FPGA built with a better semiconductor technology. However, the performance of the architecture fulfils the real-time requirements and provides the advantages of compactness and scalability for further improvements. The results presented in this section can be used for rough estimation of hardware resource utilization and evaluation of the architecture performance. For reliable comparisons with other systems and architectures, it would be necessary to use the same fabrication technology and equal amount of silicon area since smaller geometries allow packing more computation into the silicon area [13].

\section{DISCUSSION}

In this section, the window-based operation performance on the proposed architecture is compared with other currently available architectural solutions. Comprehensively comparing image processing hardware architectures is undoubtedly a complex task for a single research effort. Moreover, the lack of standard metrics increases the complexity to do reliable comparison. There are several parameters for comparison and performance evaluation of hardware architectures and systems. Some common parameters used in the literature for comparison purposes are processing speed normalized for chip area, power consumption, operation throughput, data transfer efficiency, parallel efficiency, and the product of the number of processing elements and the processing time for one frame $[7,10]$. However most of the metrics are rarely reported in the literature for architectures and systems.

\subsection{Comparison with other architectures}

The first way to make a fair comparison between the proposed architecture and other image processing architectures is within the application scope of window-based operations. The same set of algorithms should be performed on each architecture to standardize the metric for performance. Among the window-based image algorithms mapped into the systolic architecture are image convolution, template matching, and gray-level image morphology.

The comparisons made on this work are intended to be performed among several different array processor architectures. In order to do fair comparison, the architectures are compared under same conditions as

(i) they are used to deal with the same algorithm class,

(ii) the same image size is used, unless another case is established,

(iii) the execution time, clock cycles, is used as the main metric for comparison, since a lack of other metrics reported in literature.

The timings for some window-based image operators and their corresponding implementations on different hardware architectures are summarized in Table 4. In the table, the window-based image application, the machine architecture, image size, and processing time are used as main parameters for comparison $[7,8,15,16,17,18]$.
The architecture performance is similar and even better than other architectures and systems reported in the literature, as shown in Table 4. Since the architecture is not an ASIC, its performance regarding ASIC architectures is significant without sacrificing flexibility. Moreover, if the proposed architecture is implemented in an ASIC, it will provide a performance improvement of one order of magnitude.

The architecture provides a high throughput, 3.16 GOPs with power consumption of about $1.56 \mathrm{~W}$ and the processing time is independent of the window-based algorithm mapped to the architecture. Regarding power consumption, the architecture provides an improvement over two magnitude orders over Pentium processors. The power consumption of Pentium IV is about $70 \mathrm{~W}$.

\subsection{Area/performance tradeoffs}

In order to analyze the requirements of area for different configurations of the architecture, a graph of the number of FPGA hardware resources used versus a particular windowbased application was obtained. As shown in Figure 9, the hardware resources, number of LUTs, slices, and flip-flops in the FPGA depend on a particular window-based image application mapped to the FPGA. The most hardware demanding application is the convolution since it requires a multiplier per each CWP in the array to perform the operation.

From Figure 9, it can be shown that it is possible to achieve real-time performance for window-based image processing with very compact architectures. The low resource utilization allows the architecture to be implemented in compact mobile applications restricted to low-power consumption. From results presented above, it is clear that the proposed architecture obtains a very high efficiency, and it is possible to state that it provides a high computational density due to the relatively small implementation area used of the FPGA. Furthermore, since the current architecture configuration provides a computational power greater than that required for real-time performance, it is possible to reduce at some extent the hardware resources or the main clock frequency for power saving purposes.

\subsection{Multifunctional architecture}

One of the main advantages of the proposed architecture is its configurability. The architecture is flexible enough to support several variations of window-based image operators. Thus, the architecture offers the possibility of addressing different image-based applications from the system-on-a chip (SoC) perspective.

Due to its versatile, modular, and scalable design, the architecture constitutes a platform to explore more complex algorithms such as block matching for motion estimation, stereo disparity computation, and discrete cosine transformation, among others. Some of these potential applications are currently being developed. For instance, the following key aspects have to be considered in order to map a block matching algorithm into the architecture. First, the coefficients of the window mask are variable and their values are obtained of windows extracted from the reference image. Second, once a coefficient window is extracted it remains constant through 
TABLE 4: Performance comparison of the architecture with other proposed systems for window-based image processing.

\begin{tabular}{|c|c|c|c|c|}
\hline \multicolumn{5}{|c|}{ Performance benchmarks on different architectures } \\
\hline System & Architecture & Application & Image size & Timing \\
\hline TMS320C80 & Multiprocessor & $5 \times 5$ Gaussian convolution & $512 \times 512$ & $40 \mathrm{~ms}$ \\
\hline TMS320C80 & Multiprocessor & $3 \times 3$ gray-level dilation & $512 \times 512$ & $32.7 \mathrm{~ms}$ \\
\hline Splash 2 & Multi-FPGA & $3 \times 3$ median filter & $512 \times 512$ & $27 \mathrm{~ms}$ \\
\hline PDSP16488 $40 \mathrm{MHz}$ & Hardwired ASIC & $8 \times 8$ convolution & $512 \times 512$ & $6.56 \mathrm{~ms}$ \\
\hline PPIP & $64 \times 642 \mathrm{D}$ SIMD array & $5 \times 5$ Laplacian filter & $512 \times 512$ & $197 \mu \mathrm{s}$ \\
\hline PPIP & $64 \times 642 \mathrm{D}$ SIMD array & $5 \times 5$ Gaussian convolution & $512 \times 512$ & $730 \mu \mathrm{s}$ \\
\hline LSI Logic's L64240, 20 MHz & Hardwired ASIC & $8 \times 8$ convolution & $512 \times 512$ & $13.11 \mathrm{~ms}$ \\
\hline Blue wave system PCI/C6200 & DSP-based, & $3 \times 3$ convolution & $512 \times 512$ & $7.2 \mathrm{~ms}$ \\
\hline Alacron's AI-860 & I860 processor & $8 \times 8$ convolution & $512 \times 512$ & $66.1 \mathrm{~ms}$ \\
\hline UWGSP5 & DSP-based, MVP & $3 \times 3$ convolution & $512 \times 512$ & $19 \mathrm{~ms}$ \\
\hline IMAP vision & SIMD, $256 \mathrm{PE}$ & $3 \times 3$ convolution & $256 \times 256$ & $0.65 \mathrm{~ms}$ \\
\hline IMAP vision & SIMD, 256 PE & $3 \times 3$ gray-level dilation & $256 \times 256$ & $0.31 \mathrm{~ms}$ \\
\hline DECChip 21064 & Multiprocessor & $5 \times 5$ convolution & $512 \times 512$ & $220 \mathrm{~ms}$ \\
\hline MAP1000 $200 \mathrm{MHz}$ & Media processor VLIW & $7 \times 7$ convolution & $512 \times 512$ & $7.9 \mathrm{~ms}$ \\
\hline VP24000/10 & Vector computer & $8 \times 8$ template matching & $512 \times 512$ & $40 \mathrm{~ms}$ \\
\hline $500 \mathrm{MHz}$ Pentium III & Superscalar & $8 \times 8$ convolution & $512 \times 512$ & $56.5 \mathrm{~ms}$ \\
\hline Proposed architecture & Systolic & $\begin{array}{l}7 \times 7 \text { generic window-based } \\
\text { image operator }\end{array}$ & $512 \times 512$ & $8.35 \mathrm{~ms}$ \\
\hline
\end{tabular}

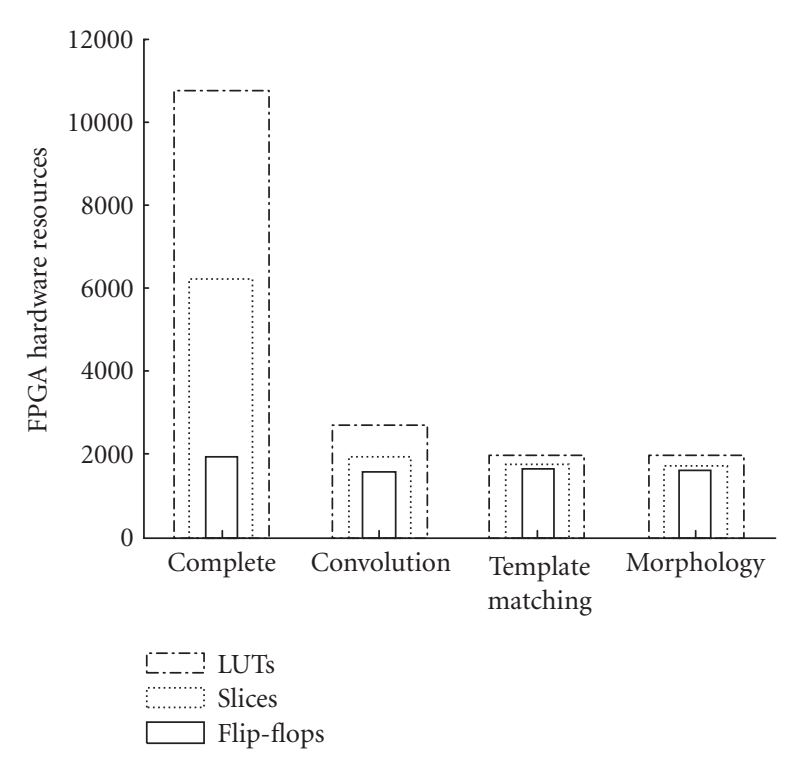

FIGURE 9: FPGA hardware resources for different architecture configurations.

the search area associated to the reference image. Similarly, the stereo matching can be formulated as a window operator such as the block matching. The difference for this case is that two static images from two points of view are considered.

\section{CONCLUSIONS}

This paper has presented an alternative and flexible FPGAbased architecture for window-based image processing under real-time constraints. A set of processors is organized under a systolic approach to form a programmable architecture. The architecture can achieve a processing rate of near 3.16 GOPs with a $60 \mathrm{MHz}$ clock frequency. The high-performance and compact hardware architecture opens new and practical possibilities to mobile machine vision systems where size and power consumption are hard constraints to overcome. The architecture was derived from a functional decomposition of window-based algorithms and operators. In order to cope with a broad class of image processing algorithms, a computational processing unit, called configurable window processor, was defined.

The programmability of the proposed architecture provides the additional advantage of being more flexible than the ASICs based on hardwired approaches. The configurable window processor developed in this research can be used and adapted to support different algorithms based on a window processing scheme. The window-based image processing algorithms addressed in this research include generic convolution, template matching, and gray-level image morphology. Moreover, some other applications suit well for the architecture such as block matching for motion estimation, stereo disparity computation, among others. In this sense, a software tool will be explored to synthesize dedicated modules for low-level image processing using as a basis the addressing mode and the parallel techniques employed in the proposed architecture.

The performance comparison with other existing architectures confirms the promising advantages of the proposed FPGA-based systolic architecture over other conventional approaches. Its performance has been evaluated for many window-based target applications with excellent results that validate the proposed high-performance architectural model. 


\section{REFERENCES}

[1] R. Jain, R. Kasturi, and B. G. Schunck, Machine Vision, McGraw-Hill, New York, NY, USA, international edition, 1995.

[2] N. Ranganathan, Ed., VLSI \& Parallel Computing for Pattern Recognition \& Artificial Intelligence, vol. 18 of Series in Machine Perception and Artificial Intelligence, World Scientific Publishing, 1995.

[3] P. A. Laplante and A. D. Stoyenko, Eds., Real-time Imaging Theory, Techniques and Applications, IEEE Press, New York, NY, USA, 1996.

[4] P. M. Athanas and A. L. Abbott, "Real-time image processing on a custom computing platform," Computer, vol. 28, no. 2, pp. 16-24, 1995.

[5] J. Silc, T. Ungerer, and B. Robic, "A survey of new research directions in microprocessors," Microprocessors and Microsystems, vol. 24, no. 4, pp. 175-190, 2000.

[6] M. K. Ratha, Computer Vision Algorithms on Reconfigurable Logic Arrays, Ph.D. Dissertation, Michigan State University, 1996.

[7] B. Bosi, G. Bois, and Y. Savaria, "Reconfigurable pipelined 2D convolvers for fast digital signal processing," IEEE Trans. VLSI Syst., vol. 7, no. 3, pp. 299-308, 1999.

[8] R. Managuli, G. York, D. Kim, and Y. Kim, "Mapping of twodimensional convolution on very long instruction word media processors for real-time performance," Journal of Electronic Imaging, vol. 9, no. 3, pp. 327-335, 2000.

[9] D. H. Ballard and C. M. Brown, Computer Vision, PrenticeHall, Englewood Cliffs, NJ, USA, 1982.

[10] D. Li, L. Jiang, and H. Kunieda, "Design optimization of vlsi array processor architecture for window image processing," IEICE Transactions on Fundamentals of Electronics, Communications and Computer Sciences, vol. E82-A, no. 8, pp. 14751484, 1999.

[11] A. Bouridane, D. Crookes, P. Donachy, K. Alotaibi, and K. Benkrid, "A high level FPGA-based abstract machine for image processing," Journal of Systems Architecture, vol. 45, no. 10, pp. 809-824, 1999.

[12] V. Moshnyaga, N. Watanabe, and K. Tamaru, "A memory efficient array architecture for real-time motion estimation," IEICE Transactions, vol. J81-D-I, no. 2, pp. 77-85, 1998.

[13] A. DeHon, "The density advantage of configurable computing," Computer, vol. 33, no. 4, pp. 41-49, 2000.

[14] P. Marchal, "Field-programmable gate arrays," Communications of the ACM, vol. 42, no. 4, pp. 57-59, 1999.

[15] M. Arias-Estrada and C. Torres-Huitzil, "Real-time field programmable gate array architecture for computer vision," Journal of Electronic Imaging, vol. 10, no. 1, pp. 289-296, 2001.

[16] B. Draper, W. Najjar, W. Böhm, et al., "Compiling and optimizing image processing algorithms for FPGA's," in International Workshop on Computer Architecture for Machine Performance (CAMP '00), pp. 240-246, Padova, Italy, September 2000.

[17] F. Lohier, Méthodologies de programmation et évaluation des processeurs de traitement de signal paralleles por le traitement d'images en temps réel, Ph.D. Dissertation, L'Universite Pierre Et Marie Curie (Paris 6), 2000.

[18] H. Singh, M.-H. Lee, G. Lu, F. J. Kurdahi, N. Bagherzadeh, and E. M. Chaves Filho, "Morphosys: an integrated reconfigurable system for data-parallel and computation-intensive applications," IEEE Trans. Comput., vol. 49, no. 5, pp. 465$481,2000$.
César Torres-Huitzil received his B.S. degree in electronic engineering from the Universidad Autónoma de Puebla (BUAP) in 1995, and his M.S. degree in electronics and his Doctor degree in computer science from the National Institute for Astrophysics, Optics and Electronics (INAOE), Puebla, Mexico, in 1998 and 2003, respectively. Since 1998, he is a Researcher of the Digital Systems Design Group in the Electronics Fac-

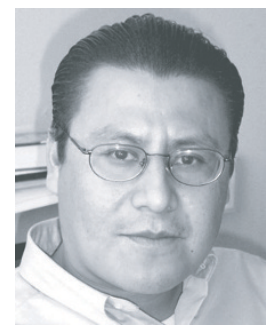
ulty, BUAP. Currently, he is a Postdoctoral Fellow at Laboratoire Lorrain de Recherche en Informatique et ses Applications (LORIA), France. His research activities include work on computer vision architectures for real-time operation, reconfigurable computing, evolutionary computation, and HDL modeling of digital systems. The current emphasis of his work is on FPGA implementation of highdensity neural networks.

Miguel Arias-Estrada received the B.Eng. degree in communications and electronics, and the M.Eng. degree in electronics from the Universidad de Guanajuato, Mexico, in 1990 and 1992, respectively, and the $\mathrm{Ph} . \mathrm{D}$. degree in electrical engineering from the Université Laval in Quebec, Canada, in 1998. From 1997 to 1998 , he was with the Electrical and Computer Engineering Department, Université Laval in Canada. Since

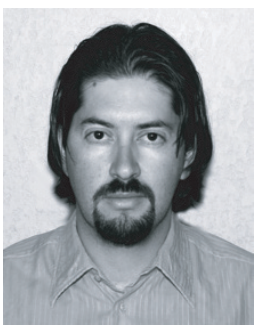
1998 he is a Researcher at the Computer Science Department, the National Institute for Astrophysics, Optics and Electronics (INAOE) in Puebla, Mexico. His current research interests are in FPGA and VLSI architectures for real-time computer vision, realtime 3D recovery, FPGA implementation of spiking neural networks, smart cameras, and evolutionary engineering applied to hardware design. 